Methods With Ethics Committee agreement (CPP-ILE-DEFRANCE III, approval date 04/02/2020), healthy volunteers were prospectively included to receive nociceptive cold stimulations at rest, without VRH (Control condition) and during VRH with HypnoVR*software (Oculus Rift ${ }^{\circledR}$ headset, Oculus VR) (VRH condition). Three intensities (Visual Analog Scale $($ VAS $)=0 / 10,2 / 10,4 / 10)$ of cold stimulations were applied 3 times each (stimulation duration: 20 seconds, separated by 1 minute) on participant's wrist with a thermode. Intensities were chosen beforehand by applying various cold intensities that subjects scored on a VAS. Maximal pain intensity and unpleasantness perception were collected after the end of each condition and then compared.

Results A total of 41 healthy volunteers were analyzed (demographic data: table 1). There is a significant decrease in pain average intensity and unpleasantness perception in VRH compared to control group $(2,46 \pm 1,59$ vs $3,66 \pm 1,84 ; \mathrm{p}<.0001$ and $3,06 \pm 2,06$ vs $2,21 \pm 1,70 ; \mathrm{p}<0,0001$ respectively) (figure 1).

Conclusions VRH (HypnoVR*) managed to decrease cold pain intensity (-33\%) and unpleasantness (-40\%) perception in healthy volunteers. These results need to be confirmed in clinical setting.

\section{OPIOID CONSUMPTION AND NEUROPATHIC PAIN IN ADJUVANT ANAESTHESIA WITH DEXMEDETOMIDINE AND LIDOCAINE}

'L Andjelković Juvan, 'A Hostnik*, 2,3| Potočnik. 'University Medical Center Ljubljana, Ljubljana, Slovenia; ${ }^{2}$ Institute of Oncology, Ljubljana, Ljubljana, Slovenia; ${ }^{3}$ Medical Faculty, University of Ljubljana, Ljubljana, Slovenia

\subsection{6/rapm-2021-ESRA.215}

Background and Aims Combination of opioid analgesics and analgesics with other mechanisms of action has synergistic analgesic effect and therefore the consumption of opioid analgesics could be decreased. It is known that dexmedetomidine and lidocaine both have opioid sparing effects, but the influence of dexmedetomidine in laparoscopic intestine resections has not been observed yet. In this study we investigated reduction in opioid consumption in patients receiving additional lidocaine or dexmedetomidine.

Methods Within the study we observed: (i) fentanyl consumption, (ii) consumption of piritramide on the first and the second postoperative day, and (iii) cognitive function before and after the operation and neuropathic pain two months after the operation. 59 participants were randomly allocated into three groups. The anaesthesia type in the control group (CG) was continuous propofol infusion and fentanyl boluses. Continuous intravenous infusion of dexmedetomidine $(0.5 \mu \mathrm{g} / \mathrm{kg} / \mathrm{h})$ and lidocaine $(1.5 \mathrm{mg} / \mathrm{kg} / \mathrm{h})$ was added to dexmedetomidine (DG) and lidocaine group (LG), respectively.

Results There was no reduction in fentanyl consumption among the groups. We noted significantly lower consumption of piritramide in LG compared with CG on the first postoperative day $(p=0.019)$, and in LG compared with DG on the second postoperative day $(p=0.003)$. There were no differences in changes in cognitive function before and after the surgery and appearance of neuropathic pain two months after the surgery.

Conclusions Lidocaine and dexmedetomidine reduced intraoperative propofol consumption but failed to decrease fentanyl demand. Lidocaine reduced piritramide consumption postoperatively.

\section{COMPARING THE EFFICACY AND SAFETY OF USG GUIDED MODIFIED PECTORAL BLOCK VS. ERECTOR SPINAE BLOCK FOR POSTOPERATIVE PAIN MANAGEMENT IN PATIENTS UNDERGOING MODIFIED RADICAL MASTECTOMY}

${ }^{1}$ A Abraham*, ${ }^{2} \mathrm{P}$ Mehta. ${ }^{1}$ Max Super Speciality Hospital, Delhi, India; ${ }^{2}$ Max Super Speciality Hospital, New Delhi, India

\subsection{6/rapm-2021-ESRA.216}

Background and Aims Breast cancer accounts for 25-32\% of all female cancers in India ${ }^{1}$. Modified radical mastectomy (MRM), a common surgical procedure, comprises 31\% of all breast surgeries performed ${ }^{2}$. A postoperative plan incorporating regional nerve blocks provide efficient analgesia, early mobilization, recovery, and prevention of chronic pain ${ }^{3}$.

This study aims to compare the efficacy and safety of modified pectoral nerve (PECS) block and Erector Spinae Plane (ESP) block for pain management in patients undergoing MRM.

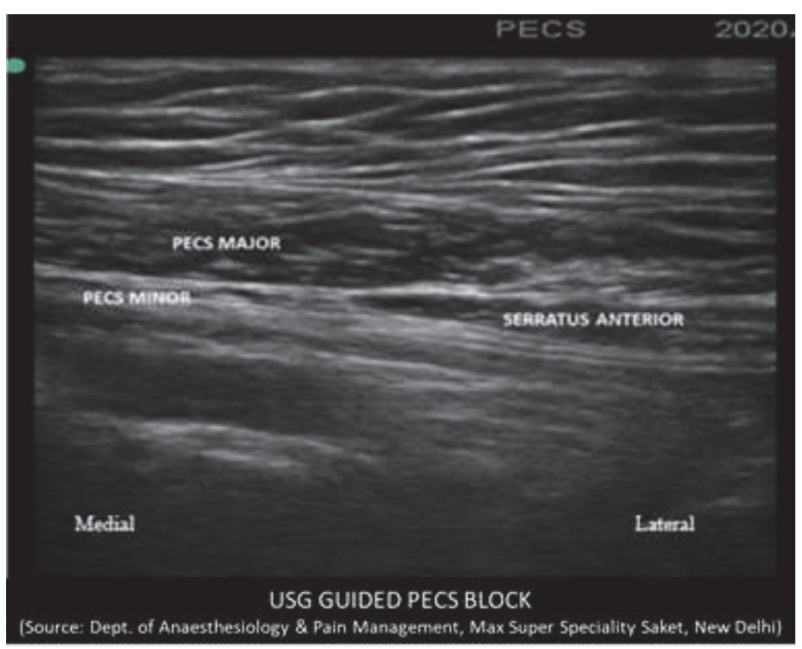

Abstract 216 Figure 1

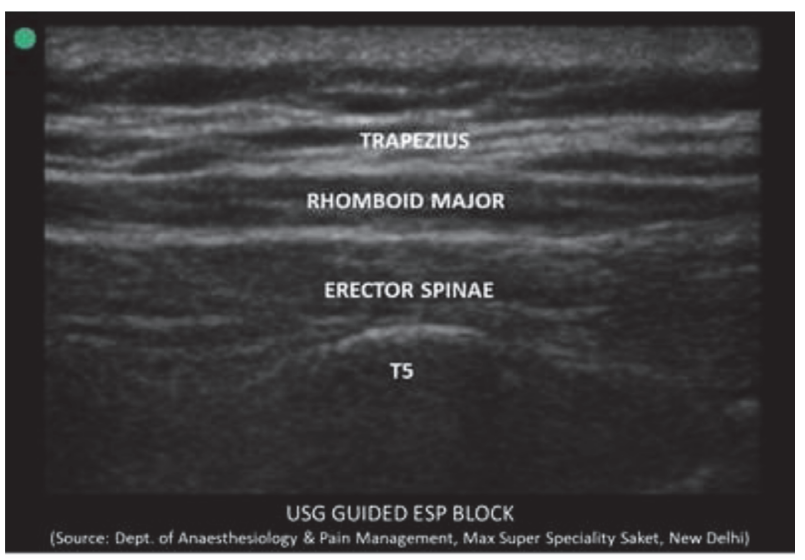

Abstract 216 Figure 2 


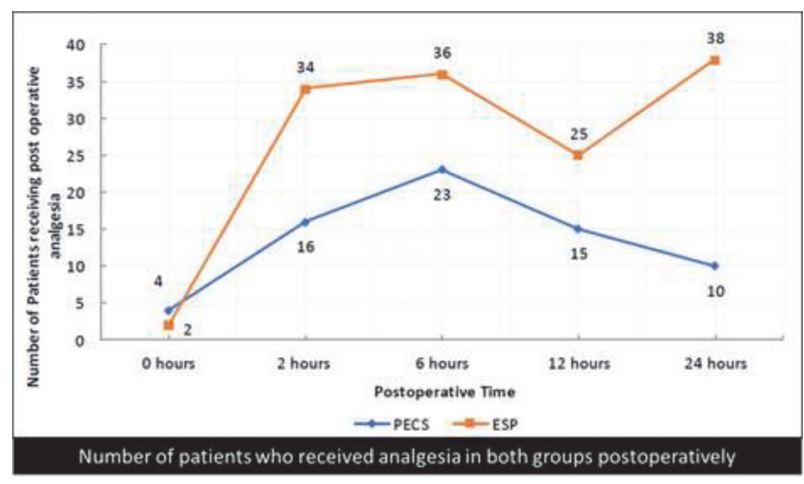

Abstract 216 Figure 3

Methods This study was registered with the Clinical Trials Registry of India (CTRI/2019/06/019656) following approval from Max Healthcare Ethics Committee.

80 adult female patients (ASA grades I\&II) undergoing modified radical mastectomy were randomly allocated into two groups to receive PECS block and ESP block with $30 \mathrm{ml}$ of $0.375 \%$ Levobupivacaine, after induction of anaesthesia. Fentanyl was administered for postoperative pain relief via PCA pump.

Outcomes such as Numerical Rating Scale (NRS) scores to assess pain, time to first rescue analgesia, intraoperative and postoperative fentanyl requirement, incidence of PONV, patient satisfaction etc. were statistically analysed.

Results Patients given modified PECS block experienced significantly better quality of analgesia and perceived the block to be more satisfactory with respect to pain relief and ability to sleep $(\mathrm{p}<0.001)$. With PECS block, patients showed significantly lower mean NRS scores at 24-hours postoperatively $(1.18 \pm 1.13$ vs $2.65 \pm 0.98)(\mathrm{p}<0.001)$ and total fentanyl consumed was considerably lesser $(61.25 \pm 41.58 \mathrm{mcg}$ vs 183.75 $\pm 51.13 \mathrm{mcg})(\mathrm{p}<0.001)$.

Conclusions We found that modified PECS block provided superior postoperative analgesia than ESP block in patients undergoing MRM without any adverse effects.

\section{EFFECTIVENESS OF TRANSVERSUS ABDOMINIS PLANE (TAP) BLOCKS IN AMBULATORY LAPAROSCOPIC AND ROBOTIC NEPHRECTOMIES}

E Lin*, J Serafin, A Vickers, B Simon, R Twersky. Memorial Sloan Kettering Cancer Center, Josie Robertson Surgery Center, New York, USA

\subsection{6/rapm-2021-ESRA.217}

Background and Aims Ultrasound-guided (USG) Transversus Abdominis Plane (TAP) blocks are fascial plane blocks widely used because of their technical ease and analgesic implications in abdominal surgery. ${ }^{1}$ However, only a few small-scale TAP studies in laparoscopic nephrectomies for tumor removal exist, none in an outpatient setting. ${ }^{2}$ We assessed the impact of TAP on pain, PONV and other clinical outcomes when included in an ERAS pathway for extended ambulatory nephrectomies.

Methods We performed a retrospective chart review of 209 ambulatory surgery patients who underwent partial laparoscopic and robotic nephrectomies using an ERAS protocol from 09/2016-12/2019. Preoperative TAP blocks were performed bilaterally with $20 \mathrm{ml}$ of $0.25 \%$ bupivacaine or ropivacaine, $50 \mathrm{mcg}$ clonidine and $2 \mathrm{mg}$ dexamethasone. We
Abstract 217 Table 1 Patient demographics and surgical details by block receipt

\begin{tabular}{|c|c|c|c|}
\hline Characteristic & No TAP, $N=102^{2}$ & TAP, $N=107^{1}$ & p-value ${ }^{2}$ \\
\hline Age & $58(49,67)$ & $61(52,68)$ & 0.3 \\
\hline Female & $43(42 \%)$ & $35(33 \%)$ & 0.2 \\
\hline ASA Score & & & 0.5 \\
\hline$\geq 3$ & $45(44 \%)$ & $53(50 \%)$ & \\
\hline BMI & $28.2(25.0,31.8)$ & $28.8(26.1,33.6)$ & 0.3 \\
\hline Apfel Score & & & 0.3 \\
\hline 0.2 & $59(58 \%)$ & $72(67 \%)$ & \\
\hline 3 & $34(33 \%)$ & $30(28 \%)$ & \\
\hline 4 & $9(8.8 \%)$ & $5(4.7 \%)$ & \\
\hline Surgery type & & & 0.5 \\
\hline Nephrectomy Partial Lap & $33(32 \%)$ & $40(37 \%)$ & \\
\hline Nephrectomy Partialw Robot & $69(68 \%)$ & $67(63 \%)$ & \\
\hline Operative Time (mins) & $146(117,180)$ & $152(116,198)$ & 0.3 \\
\hline Surgeon-administered local anesthetic & & & 0.007 \\
\hline Bupivacaine & $56(55 \%)$ & $48(45 \%)$ & \\
\hline Exparel & $8(7.8 \%)$ & $1(0.9 \%)$ & \\
\hline Exparel, Bupivacaine & $2(2.0 \%)$ & $1(0.9 \%)$ & \\
\hline None & $36(35 \%)$ & $57(53 \%)$ & \\
\hline Intraoperative MME & $47(38,57)$ & $40(30,50)$ & 0.061 \\
\hline Intraoperative use of acetaminophen & $96(94 \%)$ & $97(91 \%)$ & 0.5 \\
\hline Hours to First Ambulation & $5.7(4.2,8.2)$ & $5.4(4.6,7.7)$ & 0.8 \\
\hline Unknown & 44 & 22 & \\
\hline
\end{tabular}

assessed the association between preoperative administration USG TAP $(51 \%)$ vs. no block $(49 \%)$ on postoperative outcomes: intraoperative fentanyl, postoperative opioid consumption using morphine milligram equivalent units (MME), PONV rate and hours to first ambulation.

Results Our results demonstrated a trend toward decreased intraoperative MME requirements in the TAP group vs no TAP $(p=0.061)$. Patients receiving a TAP also required less postoperative narcotic $(p=0.043)$. After adjusting for Apfel Score, age and operative time, the need for PONV rescue medication although not significant trended $8.6 \%$ higher in those without a TAP $(95 \%$ CI $-2.7 \%, 19 \%$; $=0.11)$.

Conclusions We found evidence that US guided bilateral TAP blocks are associated with a reduced need for postoperative narcotics in ambulatory laparoscopic and robotic nephrectomies with a trend toward decreased intraoperative MME requirements. ERAS pathways should consider including TAP blocks, even for these minimally invasive surgeries.

\section{LET'S TALK ABOUT RECTUS SHEATH CATHETERS. A RETROSPECTIVE AUDIT OF ANALGESIC TECHNIQUES USED IN EMERGENCY LAPAROTOMY}

JE Bordoni*, S Siew, C Buffery, M Goulden. Liverpool University Hospitals NHS Foundation Trust, Liverpool, UK

\subsection{6/rapm-2021-ESRA.218}

Background and Aims Pain following emergency laparotomy can be difficult to manage and there is little evidence to guide best practice. We assessed current practice by reviewing analgesic modalities used within our department, including rectus sheath catheters (RSC).

Methods This audit was authorized by local committee as not requiring ethical approval. Data collection was retrospective including all patients undergoing emergency laparotomy, 